\title{
Defects in Mitochondrial Axonal Transport and Membrane Potential without Increased Reactive Oxygen Species Production in a Drosophila Model of Friedreich Ataxia
}

\author{
Yujiro Shidara ${ }^{\dagger}$ and Peter J. Hollenbeck \\ Department of Biological Sciences, Purdue University, West Lafayette, Indiana 47907
}

\begin{abstract}
Friedreich ataxia, a neurodegenerative disorder resulting from frataxin deficiency, is thought to involve progressive cellular damage from oxidative stress. In Drosophila larvae with reduced frataxin expression (DfhIR), we evaluated possible mechanisms of cellular neuropathology by quantifying mitochondrial axonal transport, membrane potential (MMP), and reactive oxygen species (ROS) production in the DfhIR versus wild-type nervous system throughout development. Although dying-back neuropathy in DfhIR larvae did not occur until late third instar, reduced MMP was already apparent at second instar in the cell bodies, axons and neuromuscular junctions (NMJs) of segmental nerves. Defects in axonal transport of mitochondria appeared late in development in distal nerve of DfhIR larvae, with retrograde movement preferentially affected. As a result, by late third instar the neuromuscular junctions (NMJs) of DfhIR larvae accumulated a higher density of mitochondria, many of which were depolarized. Notably, increased ROS production was not detected in any neuronal region or developmental stage in DfhIR larvae. However, when challenged with antimycin A, neurons did respond with a larger increase in ROS. We propose that pathology in the frataxin-deficient nervous system involves decreased MMP and ATP production followed by failures of mitochondrial transport and NMJ function.
\end{abstract}

\section{Introduction}

The health of neurons depends critically on the movement, distribution and function of their mitochondria (Hollenbeck and Saxton, 2005). This is nowhere more evident than in a range of mitochondrial dysfunctions that are manifested prominently or exclusively as diseases of the nervous system (McKenzie et al., 2004; Schapira, 2006; McFarland et al., 2007). Mitochondria supply ATP, buffer cytosolic calcium and control apoptosis, but in addition to these activities - indeed, as their consequence-mitochondria produce reactive oxygen species (ROS). Oxidative damage has been suggested as the major source of pathology for many mitochondrial diseases (Wallace, 2005), and for neurodegenerative diseases in general (Fukui and Moraes, 2008). However, neuropathology can result from other changes in mitochondrial functions, such as the disruption of mitochondrial axonal transport (Zhao et al., 2001; Baloh et al., 2007; De Vos et al., 2007), fission/fusion (Alexander et al., 2000; Delettre et al., 2000; Kijima et al., 2005; Verhoeven et al., 2006), protein import (Bauer and Neupert, 2001), and DNA replication (Schapira, 2006). To understand the

Received Jan. 31, 2010; revised April 29, 2010; accepted July 11, 2010.

This work was supported by National Institutes of Health Grant NS027073 from the National Institutes of Neurological Disorders and Stroke to P.J.H. We thank P. R. Anderson and J. P. Phillips (University of Guelph) for the generous gift of the DfhIR fly line. We thank H. C. Chang, N. Perrimon, D. Horiuchi, and A. Pilling for technical advice, W. M. Saxton for the mito-GFP fly line, and J. L. Verburg and D. M. Fekete for critical comments on this manuscript. 'Deceased Dec. 9, 2007.

This paper is dedicated to the memory of its first author, Dr. Yujiro Shidara (Jan. 9, 1972-Dec. 9, 2007)

Correspondence should be addressed to Peter J. Hollenbeck, Department of Biological Sciences, Purdue University, 915 West State Street, West Lafayette, IN 47906. E-mail: phollenb@purdue.edu.

DOI:10.1523/JNEUROSCI.0529-10.2010

Copyright $\odot 2010$ the authors $\quad 0270-6474 / 10 / 3011369-10 \$ 15.00 / 0$ mechanisms by which mitochondrial disorders cause neuronal dysfunction and death, it will be necessary to evaluate many elements of mitochondrial biology.

Friedreich ataxia (FRDA) is an autosomal recessive neurodegenerative disease caused by a GAA repeat expansion in intron 1 of the frataxin gene. The resulting deficiency in expression is associated with a dying-back neuropathy affecting sensory neurons and spinocerebellar and corticospinal motor tracts (Gibson et al., 1996; Delatycki et al., 2000; Patel and Isaya, 2001; Puccio and Koenig, 2002). Frataxin is thought to be an iron chaperone responsible for formation of iron-sulfur (Fe-S) clusters, but its deficiency is associated not only with diminished activity of FeS-containing enzymes, but also with altered defense against oxidative stress (Calabrese et al., 2005; Bencze et al., 2006). Evidence that FRDA pathology involves increased ROS levels or susceptibility to oxidative stress comes from studies of sera, tissues and isolated cells of patients (Delatycki et al., 1999; Wong et al., 1999; Emond et al., 2000; Schulz et al., 2000; Chantrel-Groussard et al., 2001; Jiralerspong et al., 2001; Piemonte et al., 2001) and from transgenic mice (Chantrel-Groussard et al., 2001; for review, see Puccio and Koenig, 2002). However, recent data from transgenic mouse models show that FRDA pathology can occur, associated with deficient activities of the electron transport chain (ETC) and other Fe-S enzymes, but without evidence of increased oxidative stress (Seznec et al., 2004, 2005).

It is difficult to predict whether a particular defect in mitochondrial function will cause ROS production to increase, decrease, or remain unchanged (Pitkanen and Robinson, 1996; Smeitink et al., 2004; for review, see Balaban et al., 2005; Fukui and Moraes, 2008). Because frataxin affects the activity of many 
Fe-S enzymes (Rotig et al., 1997; Lodi et al., 1999; Bradley et al., 2000), its deficiency should produce broader, more diffuse metabolic insufficiency than mutations in specific ETC components (Taylor and Turnbull, 2005; Schapira, 2006). However, mitochondrial functions such as membrane potential or axonal transport and distribution in axons and synapses have gone largely unexamined in the disease and its models. We have examined quantitatively the cellular neuropathology of frataxin deficiency using the frataxin knock-down Drosophila strain of Anderson (Anderson et al., 2005, 2008). We find that in the frataxindeficient nervous system, mitochondria show universally diminished membrane potential early in development, followed by temporally and geographically specific axonal transport deficits and abnormal synaptic distribution.

\section{Materials and Methods}

Genetics. A DfhIR Drosophila strain ("DfhIR1," or "UDIR1") produced by Anderson et al. (2005) expressing an inhibitory RNA that specifically inhibits frataxin expression was generously provided to us and its properties have been described in detail (Anderson et al., 2005, 2008). To observe and quantify mitochondrial location and transport in frataxindeficient larvae, we crossed the DfhIR second chromosome into the mito-GFP strain produced and described by Pilling et al. (2006) to obtain the genotype: w; P $\{$ UAS-DfIR $\} \mathrm{w}^{+} / \mathrm{SM} 2$; D42-GAL4 P $\{$ UAS-mitoGFP $\}$ e/TM3. These were crossed together to obtain mito-GFP, homozygous UAS-DfIR larvae for experiments. The control genotype was w; +; D42GAL4 P\{UAS-mitoGFP $\}$ e/TM6B, Tb Hu e. To obtain a strain in which we could observe synaptic (synaptobrevin-containing) vesicles under frataxin-deficient conditions, a similar strategy was used to cross the DfhIR chromosome into the D42-GAL4-syb-GFP background (Estes et al., 2000) to obtain the genotype: w; daG32-GAL4 P $\{$ UAS-DfIR $\} \mathrm{w}^{+}$/ CyO; P\{D42-GAL4-syb $\}$ e/TM3. The control genotype was as follows: $w ;+$; P $\{$ D42-GAL4-syb $\}$ e/TM3.

Immunoblotting and immunostaining. Whole larvae were homogenized in standard denaturing SDS-PAGE buffer and protein from 10 larvae was loaded per lane of an $8 \%$ polyacrylamide gel, electrophoresed, and transferred to PDVF. Blots were probed with mouse monoclonal antifrataxin (Millipore Bioscience Research Reagents) or rabbit anti-GFP (Clontech) and secondary IRDye-800CW goat anti-mouse or anti-rabbit (Rockland), visualized using a Gel Doc EQ epi-illumination system (Bio$\mathrm{Rad}$ ), and staining intensity of individual bands was quantified using MetaMorph software (Molecular Devices). For immunostaining of NMJs, partially dissected larvae were fixed with $4 \%$ paraformaldehyde, rinsed with PBS, washed with PBS containing $0.1 \%$ Triton X-100 and 10 $\mathrm{mg} / \mathrm{ml} \mathrm{BSA} \mathrm{(PBSTx}$ ), then stained with mouse anti-HRP, washed with PBSTx, stained with Alexa 568 goat anti-mouse (Invitrogen), mounted and observed by laser scanning confocal microscopy (LSCM) (Bio-Rad) with a $568 \mathrm{~nm}$ laser line.

Larval preparation for observing neuronal mitochondria. To observe, track and stain mitochondria in live segmental nerves, larvae were prepared by a variation on previously described methods (Hurd and Saxton, 1996; Pilling et al., 2006). Larvae at second to late third instar were pinned down and opened with an incision through the cuticle on the dorsal side, and the cuticle was peeled back slightly with forceps. Then the fat bodies were pushed gently aside, exposing the ventral ganglion and segmental nerves but leaving the axons connected to their cell bodies in the ventral ganglion and to their peripheral muscle targets. A chamber was formed around the dissected larvae by sandwiching them dorsal-side-up between a glass coverslip and a slide using dental wax as a spacer, and the chamber was filled with Schneider's insect medium (Invitrogen). Mitochondria in cell bodies, axons, and NMJs of motor neurons were observed by LSCM of mito-GFP with a $488 \mathrm{~nm}$ laser line.

Quantification of mitochondrial transmembrane potential and ROS levels. Tetrmethylrhodamine methyl ester (TMRM) (Invitrogen) was added to the dissected larval preparation at $20 \mathrm{~nm}$ in Schneider's medium for $20 \mathrm{~min}$, then replaced with $5 \mathrm{nM}$ TMRM. Quantitative fluorescence values were obtained for individual mitochondria and the adjacent cyto- plasm, and the ratio of mitochondrial to cytoplasmic fluorescence was calculated as a measure of mitochondrial membrane potential (Verburg and Hollenbeck, 2008). The superoxide indicating dye MitoSOX Red (Invitrogen) was applied at $5 \mu \mathrm{M}$ in Schneider's medium for $20 \mathrm{~min}$, then washed out with plain medium. Fluorescence values for mitochondria and adjacent cytoplasm were obtained and ratioed as just described. The superoxide indicating dye dihydroethidium (hydroethidine; Sigma) was applied at $5 \mu \mathrm{M}$ in Schneider's medium for $20 \mathrm{~min}$ and washed out before observation. As a positive control for ROS detection, the complex III inhibitor antimycin A was added to larvae at 50 or $100 \mu \mathrm{M}$ for $10 \mathrm{~min}$.

Measuring mitochondrial axonal transport. Segmental nerves were observed by LSCM in their proximal (segment A2), middle (segment A4) and distal (segment A7) regions. To track GFP-mitochondria in motor axons, a small region was bleached with the confocal laser as previously described (Pilling et al., 2006) and mitochondria moving into the bleached zone in both directions were observed. Images of mitochondria were obtained every $1 \mathrm{~s}$ for $200-250 \mathrm{~s}$ and stored as PICT files. Transport was quantified by tracking individual mitochondria using ImageJ software. The transport parameters calculated were as follows: velocity, duty cycle and flux, for both anterograde and retrograde movement. In addition, anterograde, retrograde, and pause duty cycles were calculated for both net anterograde and net retrograde moving mitochondria. Mitochondrial lengths were measured and compared in wild-type and DfhIR axons using MetaMorph software.

Determination of mitochondrial density. Mitochondrial density was calculated from LSCM images using MetaMorph software. In motor cell bodies of the ventral ganglion, the area of mito-GFP fluorescence in each cell body was divided by the area of the cell body. In NMJs, paraformaldehyde-fixed larvae were immunostained with anti-HRP to reveal the full outline of the NMJs, and the area of mito-GFP staining in each NMJ was divided by the total NMJ area. Synaptic vesicle density in NMJs was observed and quantified in identical fashion using larvae of the DfhIR; D42-syb-GFP strain.

Preparation and staining of larval sections. Larvae were fixed with paraformaldehyde, paraffin embedded, and cross sectioned at a thickness of $10 \mu \mathrm{m}$. Sections were subjected to Bodian stain, counterstained with hematoxylin and eosin, and observed and recorded using a Nikon E800 microscope with a SPOT Flex color-mosaic camera (Diagnostic Instruments). Quantitative morphometry was performed using MetaMorph software with sections from second, early third and late third instar larvae. The cortical area and number of motor cell bodies were determined at all stages, and the cross sectional area of the segmental nerve was quantified in the proximal (A2), middle (A4) and distal (A6 and A8) regions of the nerve.

\section{Results}

\section{DfhIR motor neurons show distal axonal degeneration and} cell body loss in the ventral ganglion by late third instar

To check that the DfhIR; mito-GFP strain retained the decreased frataxin expression of the original DfhIR line (Anderson et al., 2005), we performed anti-frataxin immunoblots using extracts of whole DfhIR; mito-GFP larvae at second, early third and late third instar (Fig. 1A). At all three stages, the DfhIR; mito-GFP larvae showed decreased frataxin expression compared with the wild-type mito-GFP larvae. To assess the overall neuropathology of DfhIR larvae, we examined segmental nerves (Fig. $1 B, C$ ) and motor cell bodies in the ventral ganglion (Fig. $1 D-F$ ) in sections of larvae using Bodian and hematoxylin and eosin (H\&E) staining. The DfhIR segmental nerves showed no morphological difference from wild type in any region from second to early third instar, but pathology become apparent in the distal nerve late in development. At late third instar the cross sectional area of the distal nerve had declined to $72 \%$ of wild type at segment A6 and further to $61 \%$ at $A 8$ (Fig. $1 B, C$ ). The structure of the ventral ganglion of DfhIR larvae was also indistinguishable from wild type until late third instar, when the cortical area declined to $62 \%$ of that in wild type, and the number of motor cell bodies per 

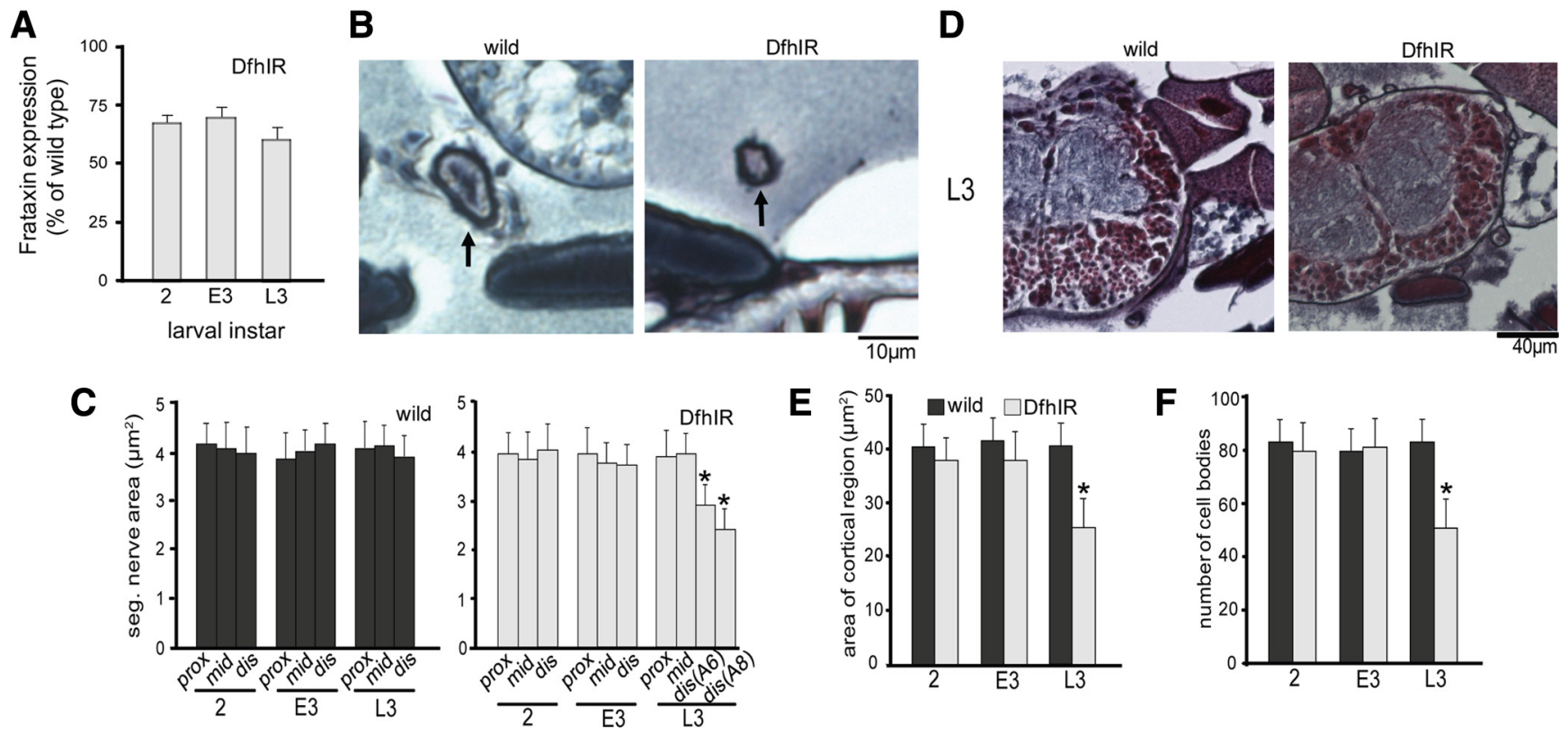

Figure 1. Axonal degeneration from the distal end and neuronal cell death in the ventral ganglion are apparent at third instar in DfhIR larvae.A, Anti-frataxin immunoblots were performed with extracts of whole larvae, at second, early third and late third instar (2, E3 and L3, respectively) quantified, and normalized for GFP content using anti-GFP. DfhIR crossed into the mito-GFP background resulted in a $30-40 \%$ reduction in whole-larval frataxin expression relative to wild type at the same stage. Bodian- and H\&E-stained paraffin sections of larvae were used to quantitate changes during development: $\boldsymbol{B}$ shows wild-type and DfhIR segmental nerve cross sections at $L 3$ in the distal axon; D shows wild-type and DfhIR ventral ganglia at L3. $\boldsymbol{C}$, Quantitive morphometry showed that in wild-type larvae, the cross sectional area of the segmental nerve did not change during development, or along the proximodistal axis at any point during development. In DfhIR larvae, the cross sectional area was significantly decreased in the distal region of the nerve (at segments A6 and A8) at L3. $\boldsymbol{E}, \boldsymbol{F}$, Morphometry of cell bodies in the ventral ganglion showed that in DfhIR larvae, both the area of the cortical region of the ganglion $(\boldsymbol{E})$ and the number of cell bodies $(\boldsymbol{F})$ were significantly decreased at $L 3$ in DfhIR relative to wild type. All error bars represent the SD, and significant differences between DfhIR and wild-type values for specific stages or regions are indicated ( ${ }^{*} p<0.05, n=40$ for all experiments).

section was reduced to $61 \%$, from a mean of just above 80 to just under 50 (Fig. $1 D-F$ ). Thus, DfhIR larvae showed dying-back neuropathy, but not until late in larval development.

To search for the underlying cellular basis of this pathology, we went on to quantify three essential features of mitochondrial activity in neurons within intact segmental nerves of these larvae: mitochondrial transport, mitochondrial transmembrane potential, and ROS production. Each of these was assessed across time and space: at three stages of larval development (second, early third and late third instar) and, where applicable, in five regions of the neurons: cell bodies in the ventral ganglion; axons in the proximal (A2), middle (A4) and distal (A7) region of the nerve, and neuromuscular junctions (NMJs).

\section{The first and most pervasive defect in DfhIR neurons is loss of mitochondrial transmembrane potential}

To compare the activity of mitochondria between wild-type and DfhIR neurons, we stained live larval preparations with a subquenching concentration of the mitochondrial dye TMRM (Farkas et al., 1989; Scaduto and Grotyohann, 1999; Verburg and Hollenbeck, 2008). The partitioning of TMRM between the cytoplasm and mitochondrial matrix is driven by the inner mitochondrial membrane potential (MMP); thus we used the ratio of mitochondrial to cytoplasmic TMRM fluorescence $\left(F_{\mathrm{m}} / F_{\mathrm{c}}\right)$ as the most sensitive and accurate available indicator of MMP. We found that in all regions of the neuron and from the earliest stage we observed, neurons in DfhIR nerves showed a significantly lower MMP than wild type (Fig. 2). In cell bodies of the ventral ganglion, $F_{\mathrm{m}} / F_{\mathrm{c}}$ was $25 \%$ lower in DfhIR neurons at second instar and declined further by late third instar (Fig. 2A,B). Mitochondria throughout the length of DfhIR axons also showed lower MMP than wild type, a difference that became more pronounced with both larval age and position along the nerve. The $F_{\mathrm{m}} / F_{\mathrm{c}}$ in the distal region of DfhIR axons was already $<70 \%$ of controls at second instar and declined further to $<50 \%$ by late third instar ( $p<0$;05 for 2 vs L3, Fig. 2D). The most extreme MMP phenotype was seen in NMJs (Fig. 2E), where the TMRM signal in DfhIR neurons declined to a mean of only $30 \%$ that of controls by late third instar (Fig. 2G). More important, DfhIR NMJs contained significant numbers of mitochondria that had no greater TMRM fluorescence than the surrounding cytoplasm and were thus completely depolarized (Fig. 2E, apparent as GFP-positive mitochondria with no yellow TMRM signal in the merged DfhIR image). Such mitochondria are virtually never seen in wild-type NMJs.

DfhIR larvae show specific defects in axonal transport of mitochondria in the distal axon, late in larval development We observed and quantified mitochondrial transport by a modification of the method of Pilling et al. (2006), photobleaching the mito-GFP in a region of segmental nerve and then following mitochondria entering that region from the proximal and distal sides. We tracked individual organelles from 250-frame sequences acquired in the proximal, middle and distal segmental nerves at second, early third and late third instar (Fig. $3 A, D$ ). From these data we calculated mitochondrial flux, duty cycle, run length, run time, and pause time for the anterograde and retrograde directions of movement in each region at each developmental stage (supplemental Table 1, available at www.jneurosci.org as supplemental material).

In wild-type segmental nerves, mitochondrial flux was greater in the anterograde than retrograde direction, and increased with larval age (Fig. 3E) while declining with distance from the cell body (Fig. $3 B$ ). Wild-type mitochondrial movement also became less directionally persistent with increased distance from the ventral ganglion: 
for anterograde-moving organelles, the anterograde duty cycle declined in the distal nerve while their retrograde and pausing duty cycles increased; the converse was true for retrograde-moving mitochondria. (Fig. 3C). However, the direction of movement was more persistent in all regions of older nerves than younger nerves (Fig. $3 F$ ).

In DfhIR segmental nerves, mitochondrial transport was similar to wild type in all parameters at second instar, but began to show small defects in the distal axons at early third instar that rose to significance at late third instar (Fig. 3G). Specifically, anterograde and retrograde flux, duty cycle, and velocity all declined significantly in distal DfhIR axons (Fig. 3H-J). The velocities of anterograde- and retrograde-moving mitochondria were almost equally affected, dropping to $77 \%$ and $73 \%$ of wild type, respectively. However, flux and duty cycle were more profoundly affected in the retrograde direction: anterograde flux dropped to $81 \%$ of wild type while retrograde flux dropped to $70 \%$, and anterograde duty cycle dropped to $83 \%$ of wild type while retrograde duty cycle dropped to $70 \%$. These diminutions of the flux and duty cycle, but not velocity, were significantly greater for retrograde movement than for anterograde movement (Fig. 3K). Thus, frataxin deficiency causes an inhibition of mitochondrial transport that is manifested in the distal axon late in larval development, and preferentially affects retrograde movement. The magnitude of these effects on mitochondrial transport is similar to that seen for hypomorphic mutant alleles of the motor proteins kinesin and dynein (Pilling et al., 2006) (our supplemental Fig. 2, supplemental Table 2, available at www.jneurosci.org as supplemental material). Finally, measurements of mitochondrial length at late third instar detected no difference between wild-type axons (mean $\pm \mathrm{SEM}=1.23 \pm 0.05 \mu \mathrm{m}$; $n=71)$ and DfhIR axons (1.37 \pm 0.14 $\mu \mathrm{m}, n=73$ ).

\section{DfhIR larvae accumulate excess depolarized mitochondria in their NMJs}

Preferential diminution of retrograde transport in the distal region would be expected to result in an abnormal accumulation of mitochondria in the NMJs of DfhIR lar-

vae. To test this, we measured the density of mitochondria in NMJs of second, early third, and late third instar larvae using mito-GFP and anti-HRP immunofluorescence signals (Fig. 4). As a baseline, we first established the developmental pattern for mitochondrial density in the cell bodies of these neurons in the ventral ganglion of live wild-type and DfhIR larvae. The projected area of mitochondria per cell body in LSCM cross sections did not
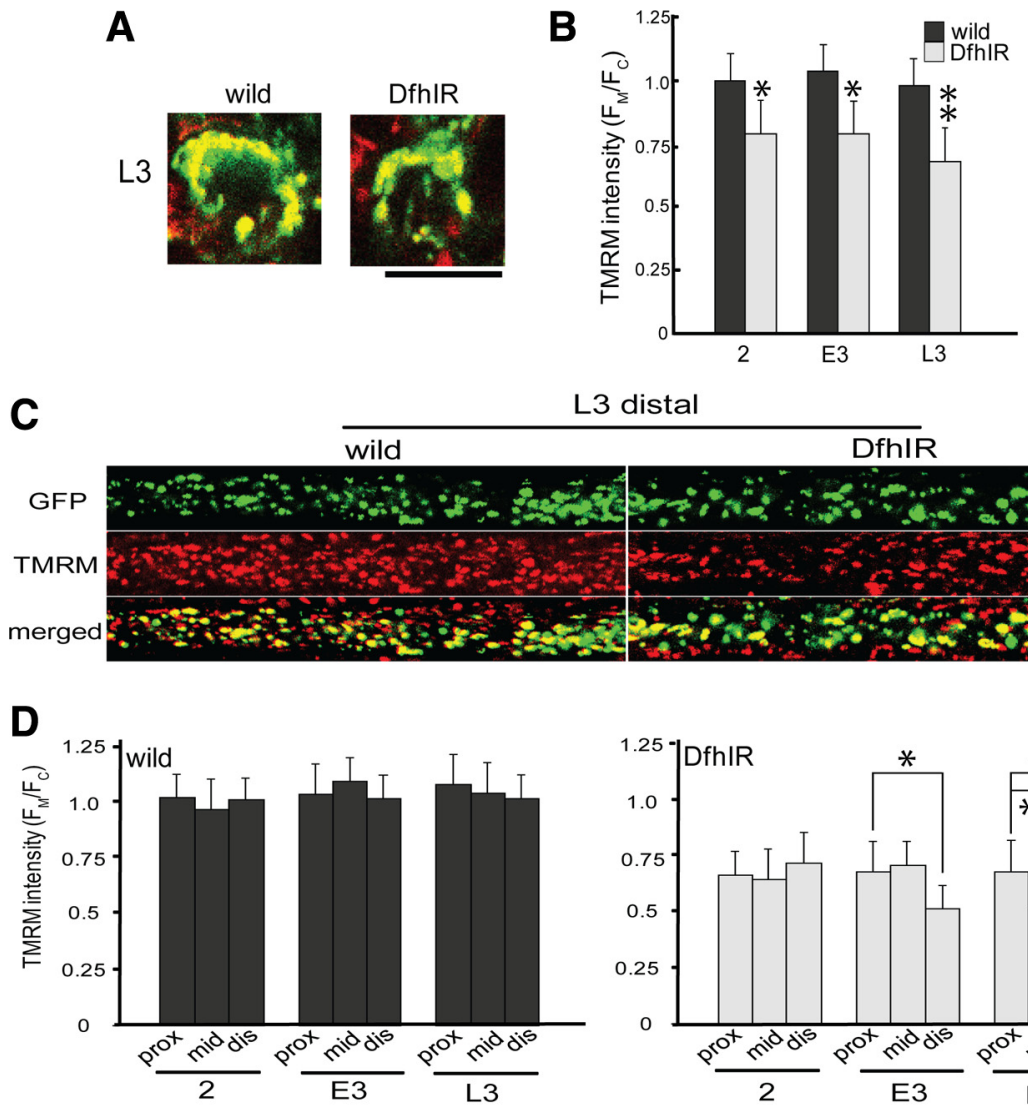

3 distal
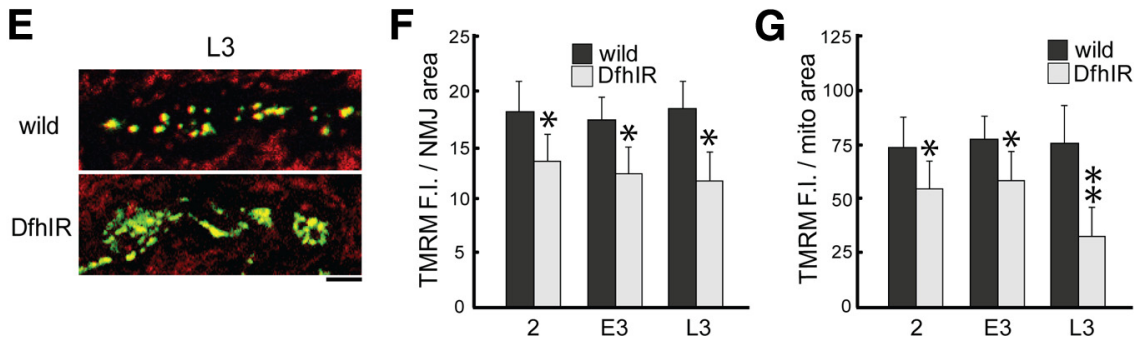

Figure 2. Mitochondrial transmembrane potential is diminished throughout the neurons of DfhIR larvae compared with wildtype. $\boldsymbol{A}, \boldsymbol{B}$, In individual cell bodies in the ventral ganglion, mito-GFP fluorescence was used to identify mitochondria $(\boldsymbol{A}$, green) and TMRM fluorescence was used to measure membrane potential (red, overlap is yellow in merged image). Note that the TMRM signal includes mitochondria in sensory axons that do not express mito-GFP and were not included in any measurements. $\boldsymbol{B}$, The ratio of mitochondrial to cytoplasmic TMRM fluorescence values $\left(F_{M} / F_{C}\right)$ was determined at second, early third, and late third instar $(2, E 3$, $L 3$, respectively), divided by the wild-type second instar mean value to normalize and plotted, showing that DfhIR cell bodies had diminished MMP throughout development relative to wild-type mito-GFP cell bodies. $\boldsymbol{C}, \boldsymbol{D}$, In segmental nerve axons, mitochondria were observed throughout development in all three regions - here, images of mitochondrial GFP fluorescence and TMRM in the distal axon at $L 3$ show the diminished MMP in DfhIR axons. $D$, The mitochondrial $F_{M} / F_{C}$ was determined in segmental nerves at each stage and axonal region and divided by the wild-type proximal second instar value to normalize. Plots of these values show that in wild-type larvae MMP did not vary with age or distance from the ventral ganglion ( $\boldsymbol{D}$, left); however, DfhIR larvae not only showed diminished MMP at all stages and axonal positions, but also showed an increasing effect with age and distance from the ventral ganglion ( $\boldsymbol{D}$, right). $\boldsymbol{E}-\boldsymbol{G}$, In neuromuscular junctions, mitochondrial TMRM fluorescence intensity per area of the NMJ had already declined relative to controls by second instar, and dropped to $<70 \%$ of control levels by late third instar $(\boldsymbol{F})$; the TMRM fluorescence per mitochondrial area declined to less than half wild type (G). Merged images of TMRM (red) and mito-GFP fluorescence at right show that at late third instar, many mitochondria in DfhIR NMJs show complete depolarization $(\boldsymbol{E})$. Scale bars: $\boldsymbol{A}, \boldsymbol{C}$, $\boldsymbol{E}, 10 \mu \mathrm{m} .{ }^{*} p<0.05,{ }^{* *} p<0.01$; error bars denote SD; $n=30$ for all TMRM measurements.

vary through development for either genotype, nor between wildtype and DfhIR larvae (Fig. 4A). In NMJs of fixed wild-type larvae, we found that the mitochondrial content and total area of NMJs both increased modestly through development, yielding no increase in mitochondrial density (Fig. 4D-F). However, in DfhIR NMJs, the total NMJ area increased during development identically to wild type, but the NMJ mitochondrial content increased 
A

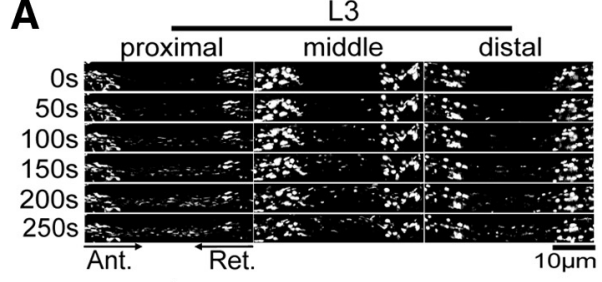

B

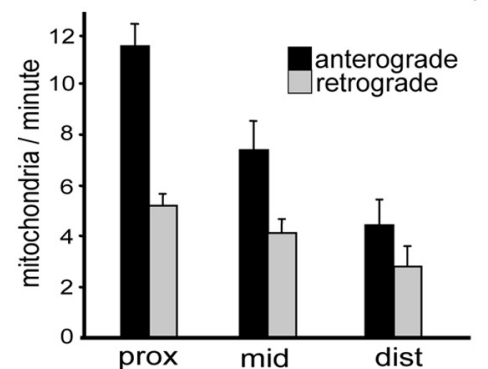

C

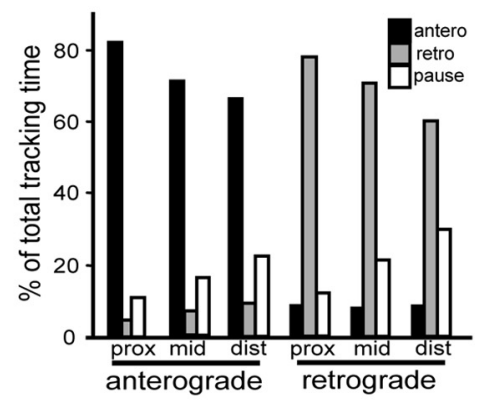

G

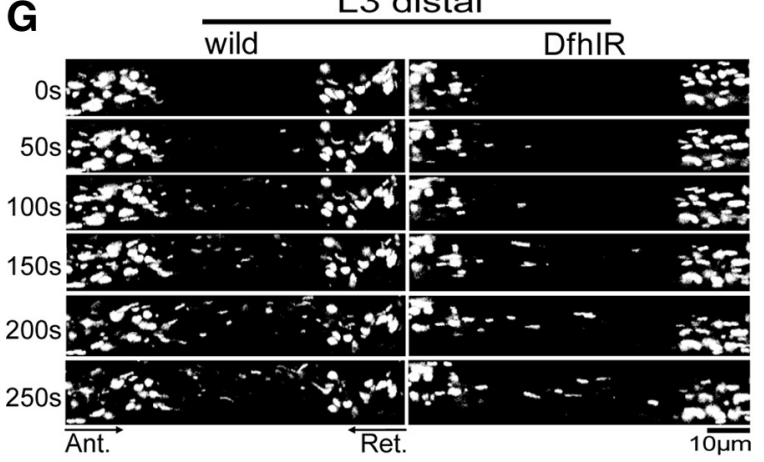

I

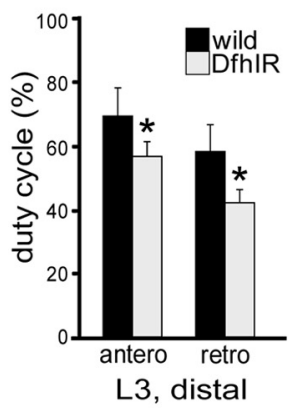

J

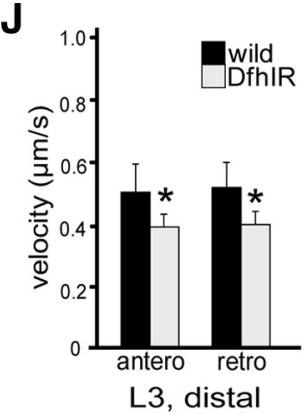

$\mathbf{H}$
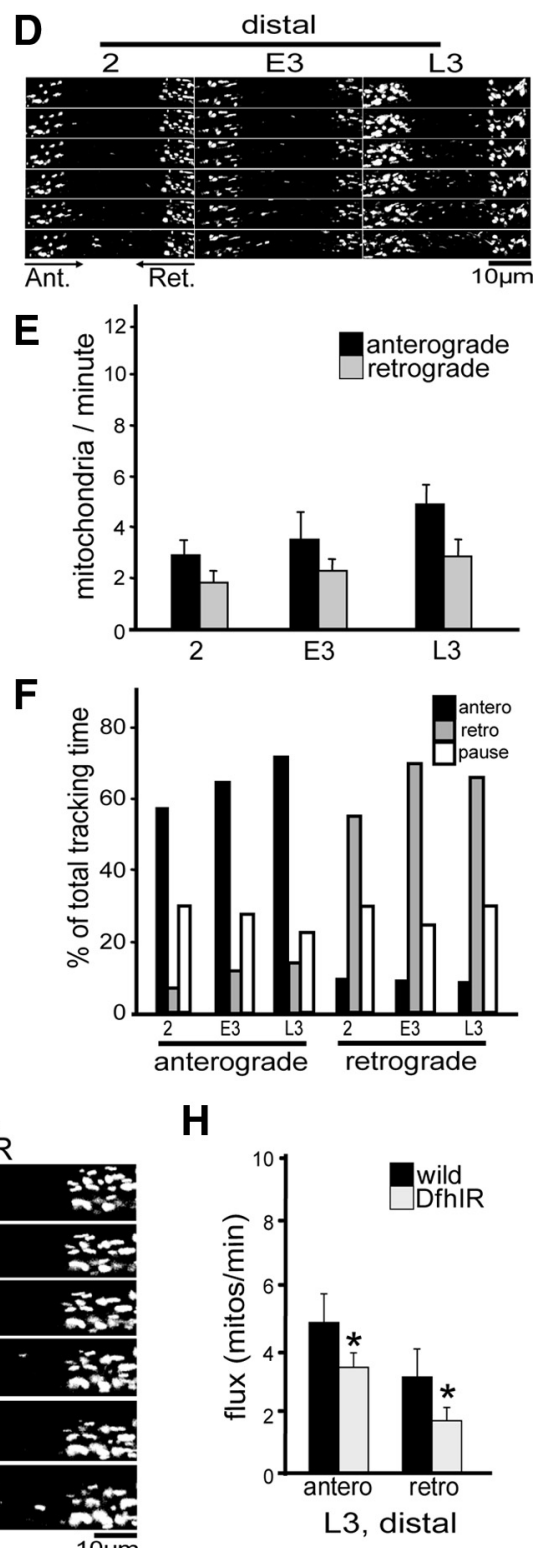

E

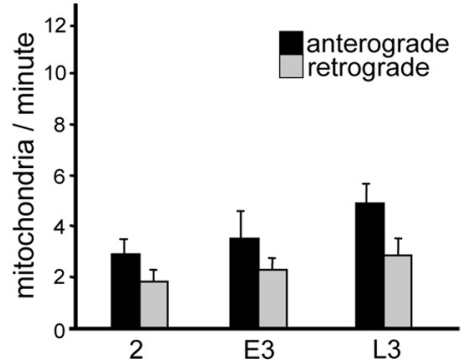

$\mathbf{F}$

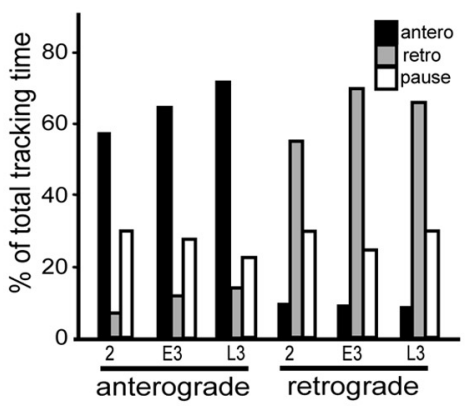

H

K

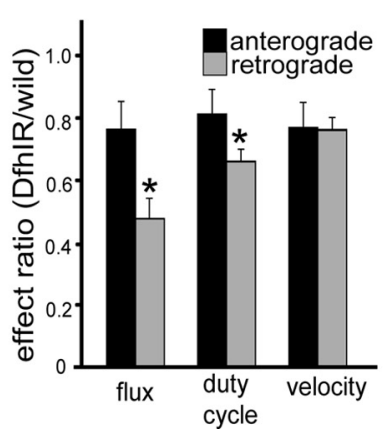

Figure 3. In segmental nerve axons of DfhIRlarvae, mitochondrial flux, transport duty cycle and velocity are diminished compared with wild type at late third instar. Mitochondrial movements were tracked in segmental nerves of second, early third and late third instar larvae, in the proximal, middle and distal region of the nerves. All images are oriented with the cell bodies (ventral ganglion) to the left, $s 0$ that anterograde transport is from left to right. $A$ shows images of mitochondria in a wild-type late third instar larva moving into a bleached region in both directions in all three regions of the nerve. $\boldsymbol{B}, \boldsymbol{C}$, Both the flux of mitochondria $(\boldsymbol{B})$ and their duty cycle $(\boldsymbol{C})$ decline from the proximal to distal axons. $\boldsymbol{D}$ shows mitochondria moving into a bleached region of the distal nerve of normal larvae in both directions, at three stages of development. $\boldsymbol{E}, \boldsymbol{F}$, In axons of the distal nerve, both the flux of mitochondria $(\boldsymbol{E})$ and their duty cycle $(\boldsymbol{F})$ increase during development from second to late third instar. $\mathbf{G}$ compares mitochondrial movements between wild-type and DfhIR larvae at late third instar in the distal region. $\boldsymbol{H}-\boldsymbol{K}, \mathbf{I n}$ DfhIR larvae, mitochondrial flux $(\boldsymbol{H})$, duty cycle $(\boldsymbol{I})$ and velocity $(\boldsymbol{J})$ are significantly reduced in both directions, but retrograde flux and duty cycle are more strongly affected than anterograde $(\boldsymbol{K})$. Scale bars, $10 \mu \mathrm{m} .{ }^{*} p<0.05, n \geq$ 30 for each experiment; error bars represent the SD.

significantly more than did wild type at late third instar, resulting in a 1.3 -fold higher mitochondrial density in DfhIR NMJs (Fig. $4 F$ ). Thus, by late third instar, frataxin deficiency leads an accumulation of excess mitochondria in NMJs, many of which are partially or completely depolarized, as shown in Figure $2 E-G$.

To ascertain whether the accumulation of organelles in the NMJs was specific to mitochondria or more general, we also quantified the density of synaptic (synaptobrevincontaining) vesicles using an n-syb-GFP; DfhIR strain (Estes et al., 2000). Using n-syb-GFP fluorescence and anti-HRP immunostaining in fixed larvae (Fig. 5A), we found that the synaptic vesicle density of NMJs rose similarly through development in both wild-type and DfhIR larvae, but that by late third instar the DfhIR NMJs accumulated synaptic vesicles to a 1.3-fold excess over wild type (Fig. $5 B$ ), very similar to that seen for mitochondria.

DfhIR neurons do not produce higher levels of ROS, but are more susceptible to complex III inhibition late in development

The body of work on Friedreich ataxia and frataxin deficiency suggests that a likely component of the pathology is generation of excess ROS (Calabrese et al., 2005; Bencze et al., 2006). We used fluorescent probes for superoxide to quantify the level of ROS in the motor neurons of live larvae. To detect superoxide levels in mitochondria, we treated larval preparations with MitoSOX red, a fluorescent lipophilic cation that, like TMRM, partitions into mitochondria and has a fluorescence intensity that increases with superoxide concentration (Mukhopadhyay et al., 2007). Using the mito-GFP signal to identify mitochondrial pixels in the confocal images, we determined MitoSOX fluorescence in cell bodies, axons and NMJs throughout larval development (Fig. 6). In wild-type larvae, in all regions of the neurons, ROS levels remained relatively constant during development, but they responded sharply to the application of the complex III inhibitor antimycin A (Staniek and Nohl, 2000; Chen et al., 2003), demonstrating the capacity of the probe to detect increases in [ROS] (Fig. 6B, D,F).

In cell bodies in the ventral ganglion, elevated mitochondrial ROS levels were not detected in DfhIR larvae relative to wild type at any stage in development. However, at late third instar, the DfhIR cell bodies did respond to a $100 \mu \mathrm{M}$ antimycin A challenge with a $40 \%$ greater increase in ROS than did wild type (Fig. 6B). 

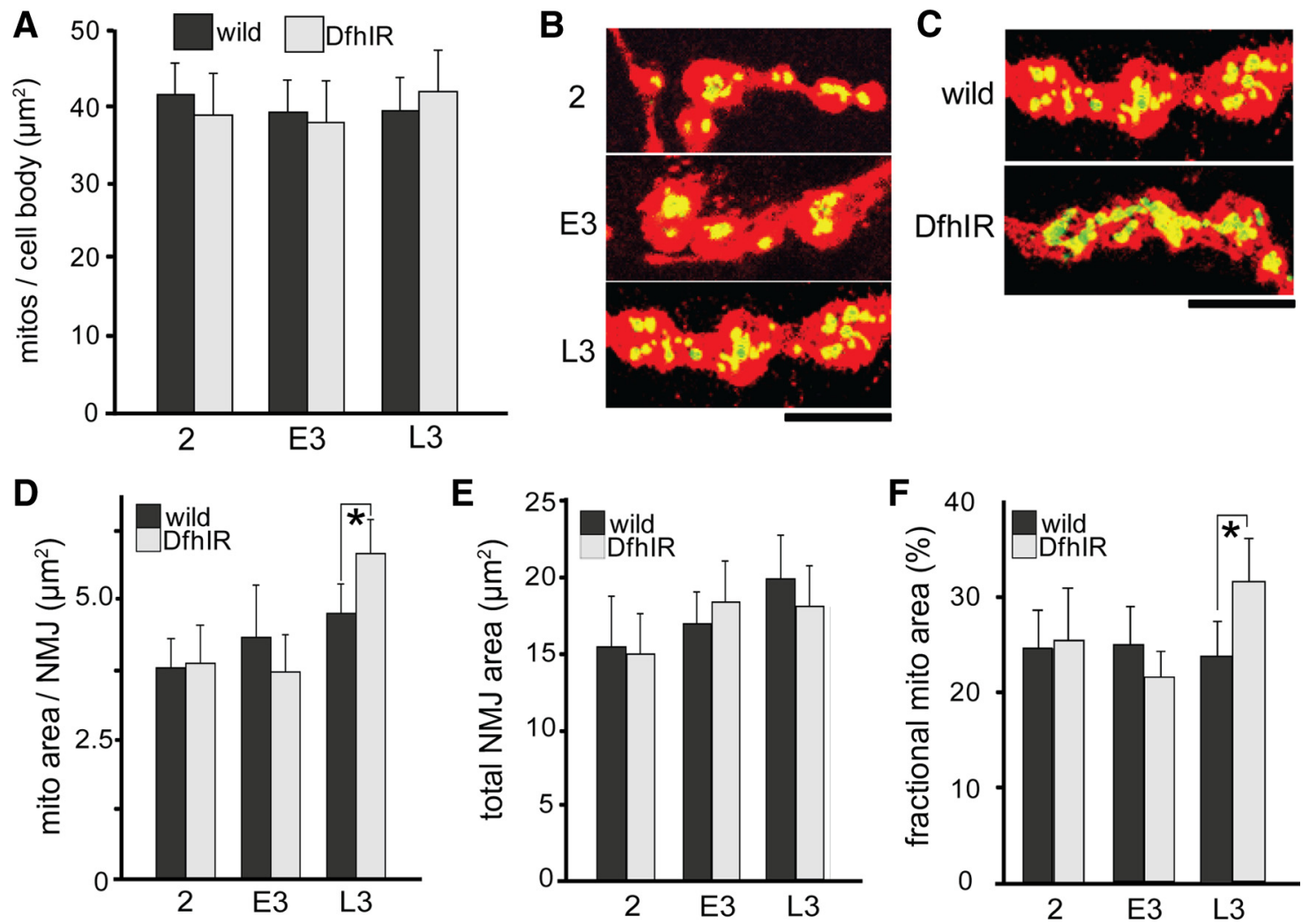

Figure 4. DfhIR larvae show an abnormal accumulation of mitochondria in the NMJs by late third instar. $A$, The density of mitochondria in motor cell bodies of the ventral ganglion, determined as the ratio of mito-GFP area to neuronal cell body area, remains nearly constant during development and is the same in wild-type and DfhIR larvae. $\boldsymbol{B}$, A merged image of HRP immunostaining (red) and mito-GFP (green, yellow in overlap with HRP) shows wild-type NMJs of second, early third, and late third instar larvae. C, A merged image of mito-GFP and HRP immunostaining shows that in late third instar larvae, NMJs of DfhIR larvae have a higher mitochondrial density than those of wild type. $\boldsymbol{D}-\boldsymbol{F}$, Quantitation of the area of mito-GFP signal per NMJ (D), total NMJ area $(\boldsymbol{E})$, and the fraction of the NMJ occupied by mitochondria $(\boldsymbol{F})$ for wild-type and DfhIR larval NMJs from second through late third instar shows the increases in both mitochondrial and total NMJ area with development, and confirms the excessive mitochondrial density of late third instar DfhIR NMJs. Scale bar, $5 \mu \mathrm{m} .{ }^{*} p<0.05, n=40$ for all experiments; error bars represent the SD.

This pattern also occurred in axonal mitochondria: DfhIR axons showed no greater ROS levels than wild type at any region or stage of development, but in response to antimycin A, they showed significantly greater mitochondrial ROS increases in the middle axons at late third instar (125\% of wild type), and in distal axons at early and late third instar $(145 \%$ and $155 \%$, respectively) (Fig. 6C,D). In NMJs, DfhIR larvae also did not show increased ROS compared with wild type until late third instar, when DfhIR NMJs showed a much greater ROS response to antimycin A challenge $(160 \%)$ than did wild type (Fig. $6 G-I$ ). Very similar results were obtained using dihydroethidium to detect total cytoplasmic superoxide: in unperturbed preparations, wild type and DfhIR did not have detectably different ROS levels at any stage of development or region of the neurons, but an antimycin challenge elicited higher ROS levels in cytoplasm of DfhIR cell bodies (170\%), distal axons (175\%) and NMJs (160\%) at late third instar only (supplemental Fig. 3, available at www.jneurosci.org as supplemental material). Thus, in the unperturbed larval nervous system frataxin deficiency did not result in detectably higher ROS levels, but it did produce hypersensitivity to complex III inhibition late in development.
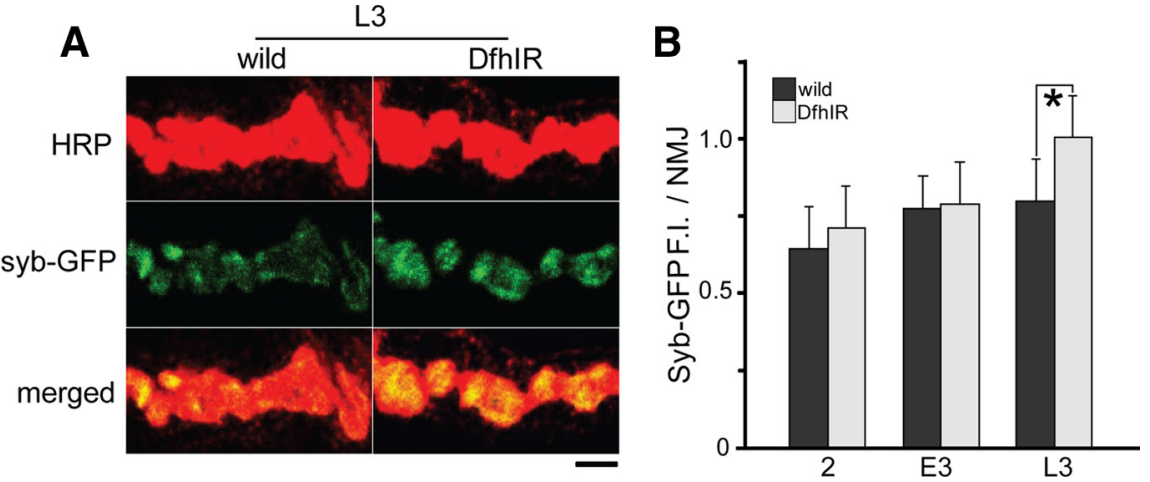

Figure 5. DfhIR larvae accumulate excess synaptobrevin-containing vesicles in their synaptic NMJs at third instar compared with controls. $A$, Synaptic (synaptobrevin-containing) vesicles in motor neurons were visualized by D42-syb-GFP (green) and NMJs were counterstained with anti-HRP antibody (red, overlap with green shown as yellow); late third instar is shown here. $\boldsymbol{B}$, Synaptic vesicle densities were determined as the ratio of the number of syb-GFP pixels to the number of HRP pixels at second, early third and late third instar. Late third instar DfhIR larvae show increased density of synaptic vesicles in their NMJs relative to wild type. All error bars represent the SD and significant differences between DfhIR and wild-type NMJs are indicated $\left({ }^{*} p<0.05, n=40\right.$ for all experiments). Scale bar, $2.5 \mu \mathrm{m}$.

\section{Discussion}

Many defects in mitochondrial function are associated with diseases of the nervous system (McKenzie et al., 2004; Wallace, 2005; Schapira, 2006; McFarland et al., 2007), but in most cases we understand neither which of these potential defects underlie the cellular neuropathology, nor their temporal and causal relationships. In this study we chose a model mitochondrial 
A
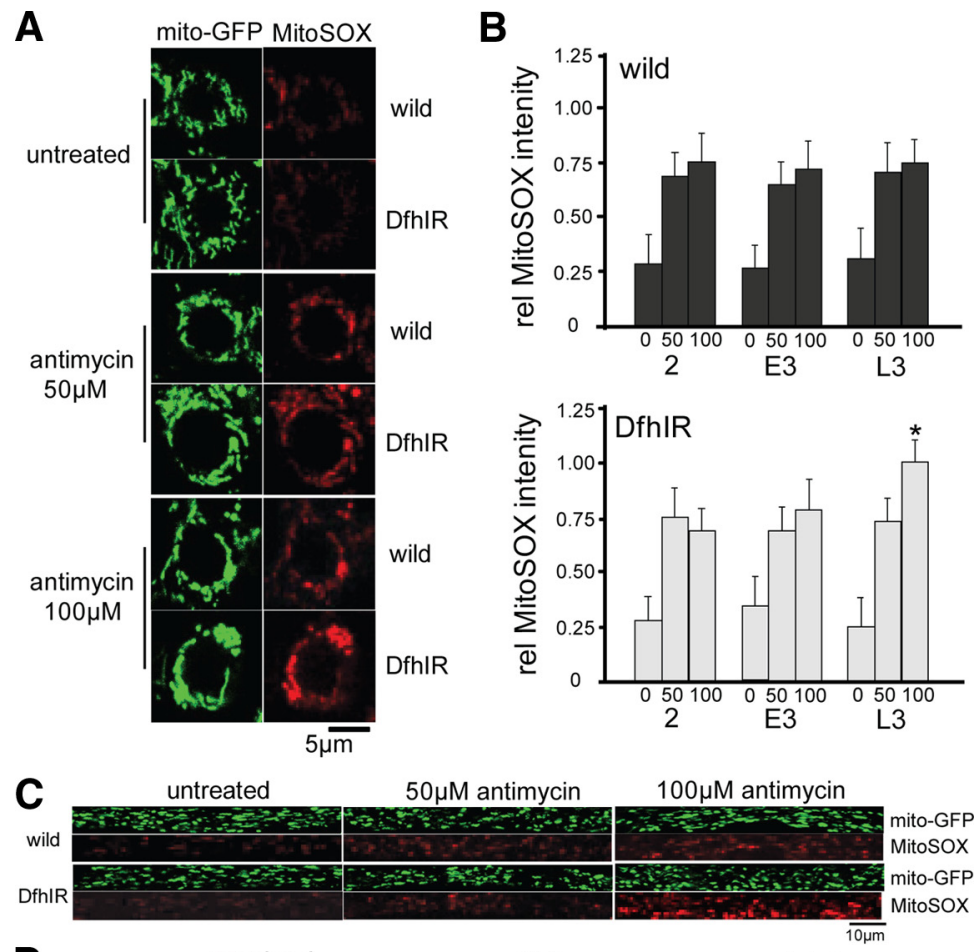

D

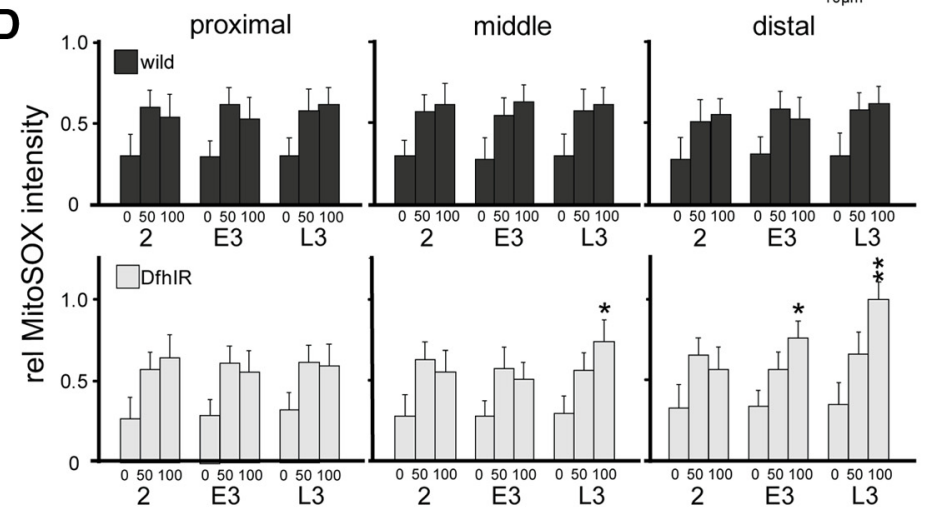

E

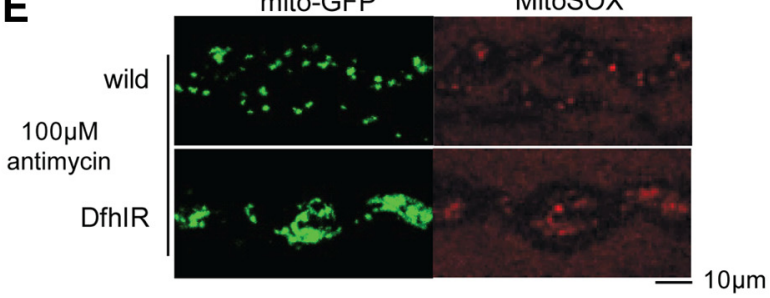

$\mathbf{F}$
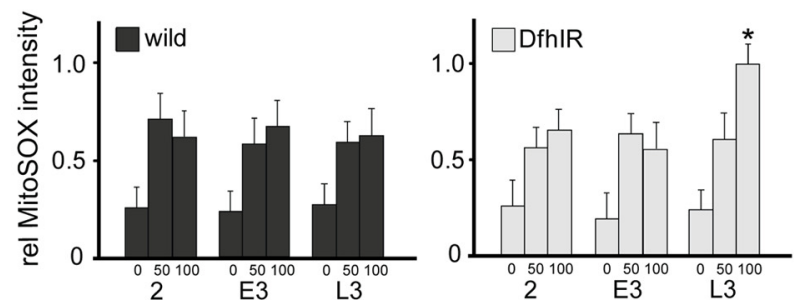

Figure 6. DfhIR neurons do not produce intrinsically higher levels of ROS, but are more susceptible to treatment with the complex III inhibitor antimycin. $\boldsymbol{A}-\boldsymbol{F}$, Fluorescence intensities of mitoGFP (green) and MitoSOX (red) were used to determine relative levels of ROS production in cell bodies in the ventral ganglion $(\boldsymbol{A}, \boldsymbol{B})$, axons $(\boldsymbol{C}, \boldsymbol{D})$, and NMJs $(\boldsymbol{E}, \boldsymbol{F})$ of DfhIR and wild-type neurons, with and without antimycin treatment, and throughout larval development. Neither wild-type nor DfhIR cell bodies showed increased ROS during development, and DfhIR cell bodies ( $\boldsymbol{B}$, top histogram) did not show higher ROS levels than wild type ( $\boldsymbol{B}$, bottom histogram). Both 50 and $100 \mu \mathrm{m}$ antimycin treatments elicited higher ROS levels in wild-type and DfhIR cell bodies at all developmental stages, but at late third instar, DfhIR cell bodies responded to antimycin with higher ROS levels than did wild type $(\boldsymbol{B})$. In axons $(\boldsymbol{C})$, there were also no significant differences between DfhIR and wild-type ROS levels in any region of the nerve or disorder, frataxin deficiency, and dissected the cellular basis of its pathology in the live, intact nervous system of Drosophila larvae. We found evidence for a developmentally ordered, counterintuitive series of defects in mitochondrial function that preceded dying-back neuropathy and did not involve apparent excess ROS production.

\section{Frataxin deficiency depresses \\ MMP early and widely in the \\ larval nervous system}

The first abnormality of mitochondrial function was also the most pervasive: DfhIR mitochondria showed significantly reduced MMP from the cell bodies throughout the axons and into the NMJs, and from second instar to late third instar. This is consistent with frataxin's proposed major role of supporting the construction and maintenance of Fe-S cluster-containing enzymes (Puccio and Koenig, 2002). Since the Fe-S components of the ETC complexes are absolutely essential for oxidative phosphorylation, DfhIR neurons are probably manifesting a general deficiency in the ETC activity that generates the MMP. This establishes, at the whole-mitochondrial level, a basis for evidence for reduced metabolic enzyme activity in Friedreich ataxia patient material (Rotig et al., 1997; Bradley et al., 2000), animal models (Puccio et al., 2001; Seznec et al., 2004; Anderson et al., 2005; $\mathrm{Lu}$ and Cortopassi, 2007), and yeast or cultured cell studies (Wilson and Roof, 1997; Stehling et al., 2004). However, since the energy of the MMP is harnessed for many functions of mitochondria, its deficiency here raises the possibility that the cellular neuropathology involves additional elements in addition to energy metabolism. Thus, mitochondrial calcium uptake, transport of other ions and metabolites, protein import, fission and fusion, all of which rely on the MMP, are potential targets in frataxin-deficient neurons. It is unclear from first principles which of these functions is most likely to be compromised by partial depolarization of mi-

$\leftarrow$

time in development, but DfhIR axons showed higher ROS levels in response to antimycin in the middle region of the axons at late third instar, and in the distal region at early and late third instar (D). There was also no difference between ROS levels in DfhIR and wild-type NMJs through development, but DfhIR NMJs at late third instar responded to antimycin with higher ROS levels than wild type $(\boldsymbol{E}, \boldsymbol{F})$. For quantification, all error bars represent the SD and significant differences between DfhlR and wild-type values are indicated $\left({ }^{*} p<0.05\right.$, ${ }^{* *} p<0.01, n=40$ for all experiments). 
tochondria, but our data (discussed below) indicate that axonal transport is affected.

MMP in the DfhIR nervous system was not only reduced relative to wild type in all locations and stages of development, but also showed a significant further decline in the middle and distal axons and NMJs late in larval development (Fig. 2), around the time of the dying-back neuropathy. This could reflect the challenge of supporting Fe-S enzyme biosynthesis at the greatest distances from the cell body and nuclear genome, and probably in turn underlies the specific deficits in axonal transport that we observed at the same stage and locations.

\section{Axonal transport deficits and accumulation of depolarized mitochondria are concurrent with axonal neuropathy}

The axon relies on the transport of mitochondria (Hollenbeck and Saxton, 2005; Chang and Reynolds, 2006), and mutations in motor proteins can cause neurodegeneration (Saxton et al., 1991; Hurd and Saxton, 1996; Hurd et al., 1996; Gindhart et al., 1998; Yonekawa et al., 1998; Zhao et al., 2001; Hafezparast et al., 2003; Hirokawa and Takemura, 2004; Pilling et al., 2006). But neuropathologies are also associated with transport defects in disorders where motor proteins are not mutated, such as amyotrophic lateral sclerosis (Bruijn et al., 2004; De Vos et al., 2007), Huntington's disease (Gauthier et al., 2004) and Charcot-Marie-Tooth disease type 2A (Baloh et al., 2007). Of particular relevance here, a mouse model of hereditary spastic paraplegia (paraplegindeficient) showed accumulation of abnormal mitochondria in synaptic terminals well before distal axonopathy or gross symptoms were detected (Ferreirinha et al., 2004). In addition, mutations in proteins such as Milton (Stowers et al., 2002; Glater et al., 2006), Miro (Guo et al., 2005), or Drp1 (Verstreken et al., 2005), whose primary effect is to deny mitochondrial supply to the distal axon, result in cellular and organismal neuropathies.

In frataxin-deficient nerves, we found that mitochondrial transport was reduced in the distal axons at late third instar, the same stage and location as the steepest declines in MMP. However, the nature of the transport deficit was counterintuitive: its net effect was not failure to deliver mitochondria to the NMJs, but failure to retrieve them. Nonetheless, because the excess mitochondria accumulating in the NMJs were profoundly depolarized, the distal axon and synapse were denied adequate mitochondrial function. Since the loss of MMP in DfhIR nerves began well before the transport deficit or dying-back of axons, and because further depolarization in distal axons was matched by transport deficits at the same stage and location, we suggest that the primary failure in the DfhIR neurons was inadequate local production of ATP to support transport. Diminished ATP production has also been reported in FRDA patient tissues (Lodi et al., 1999). The loss of MMP could also affect axonal mitochondrial transport at an indirect, regulatory manner, perhaps through changes in MMP-dependent calcium homeostasis or altered ATP-dependent signaling. In either case, we suggest that the more profound effect of DfhIR physiology on mitochondrial duty cycle than on velocity reflects the fact that velocity of movement is an intrinsic property of motor proteins, which tend to be "on" or "off" without an intermediate, "hobbled" state.

The accumulation of not just mitochondria but also vesicles in NMJs (Fig. 5) indicates that the deficit in transport was a broad one, and in each case was retrograde-selective. This is not the first report of a neurodegenerative phenotype associated with selective defects in the retrograde transport machinery (LaMonte et al., 2002; Hafezparast et al., 2003; Puls et al., 2003; Edgar et al., 2004; Horiuchi et al., 2005; Pilling et al., 2006). This selectivity could result from a differential sensitivity of the retrograde transport machinery to ATP concentration, or, again, to calcium- or ATP-dependent signaling effects. It is worth noting that mild mutant alleles of kinesin heavy chain and dynein have effects of similar magnitude to those we observed for DfhIR (supplemental Fig. 2, available at www.jneurosci.org as supplemental material) (Pilling et al., 2006). A failure of retrograde transport might also contribute to cell body loss and dying-back neuropathy by restricting the retrograde flow of trophic support (McCabe et al., 2003; Keshishian and Kim, 2004; Sanyal et al., 2004; Liu and Butow, 2006; Marqués and Zhang, 2006; Ibáñez, 2007). Altogether, our data suggest that the transport deficit is an effect of deficient mitochondrial metabolism that precedes and may contribute to dying-back neuropathy.

\section{ROS production and sensitivity}

Contrary to our expectations, in unperturbed DfhIR larvae we were unable to detect any increase in ROS levels in any part of the nervous system at any point in development. The efficiency of the superoxide dismutase, glutathione peroxidase and peroxyredoxin systems, along with small molecule ROS scavengers (Halliwell, 2006), ensures that the net ROS released to the cytoplasm amounts to a very small fraction of the total generated within mitochondria (Balaban et al., 2005) —which is itself probably 1-2 orders of magnitude smaller than often cited (Staniek and Nohl, 2000; St-Pierre et al., 2002). Thus, to ensure that we could detect any increase in ROS production, we measured both cytoplasmic and matrix ROS levels, and both methods were easily able to detect the increase in ROS production that accompanied inhibition of complex III by antimycin A. While studies of yeast, vertebrate cell lines, animal models and patient material indicate that the pathology of FRDA/frataxin deficiency is associated with increased susceptibility to oxidative damage and/or increased ROS production (cited above), animal models have also recapitulated FRDA neuropathology without evidence of increased oxidative damage (Seznec et al., 2004).

In Drosophila, studies of three different frataxin-deficient models have provided some insight. Studies in one frataxindeficient RNAi-based model showed an organismal phenotype that included reduced life span and climbing activity, and an excessive decline, in response to hyperoxia, of aconitase but not succinate dehydrogenase activity (Llorens et al., 2007). In addition, Runko et al. (2008) have shown that overexpression of frataxin in Drosophila increases both life span and resistance to some oxidative stresses. Using a model expressing one copy of the same DfhIR used in this study, Anderson et al. (2005) showed that overexpression of the ROS-scavenging enzymes superoxide dismutase or catalase did not rescue failed eclosion. However, they subsequently determined that ectopic expression of catalase, but not superoxide dismutase, rescued mitochondrial aconitase activity and life span in DfhIR animals (Anderson et al., 2008). Our studies showed that DfhIR larvae do produce excessive ROS when challenged by complex III inhibition, but only late in development, at a time when loss of distal axons and cell bodies is already occurring.

We propose that the cellular neuropathology of FRDA involves the following series of events: First, long before any gross organismal phenotype is apparent, a loss of Fe-S enzymes leads to deficient activity of the ETC, which is manifested as diminished MMP. This gives rise to diverse possible downstream effects, including reduced ATP production, calcium homeostasis and mitochondrial maintenance, particularly in the distal axon. These in turn result in an abnormal distribution of distal mitochondria, 
inadequate synaptic function, and neuronal degeneration. An overall inhibition of retrograde retrieval or trophic factors may also contribute to neuronal death. Since low MMP is likely to produce many different downstream effects, we suggest that FRDA be approached at the molecular and cellular level as a multihit phenotype (Brookes et al., 2004). That is, several fundamental defects, in a causative chain or in parallel, exacerbated by an impaired neuronal response during periods of increased environmental and oxidative stress, give rise over time to the neurodegenerative phenotype. Finally, since it is clear that ROS are essential signaling molecules in the nervous system (Floyd, 1999; Maher and Schubert, 2000; Floyd et al., 2002), any consideration of abnormal ROS levels should recognize that their major effects may occur through the subversion of signaling pathways, in addition to nonspecific oxidative damage.

\section{References}

Alexander C, Votruba M, Pesch UE, Thiselton DL, Mayer S, Moore A, Rodriguez M, Kellner U, Leo-Kottler B, Auburger G, Bhattacharya SS, Wissinger B (2000) OPA1, encoding a dynamin-related GTPase, is mutated in autosomal dominant optic atrophy linked to chromosome 3q28. Nat Genet 26:211-215.

Anderson PR, Kirby K, Hilliker AJ, Phillips JP (2005) RNAi-mediated suppression of the mitochondrial iron chaperone, frataxin, in Drosophila. Hum Mol Genet 14:3397-3405.

Anderson PR, Kirby K, Orr WC, Hilliker AJ, Phillips JP (2008) Hydrogen peroxide scavenging rescues frataxin deficiency in a Drosophila model of Friedreich's ataxia. Proc Natl Acad Sci U S A 105:611-616.

Balaban RS, Nemoto S, Finkel T (2005) Mitochondria, oxidants, and aging. Cell 120:483-495.

Baloh RH, Schmidt RE, Pestronk A, Milbrandt J (2007) Altered axonal mitochondrial transport in the pathogenesis of Charcot-Marie-Tooth disease from mitofusin 2 mutations. J Neurosci 27:422-430.

Bauer MF, Neupert W (2001) Import of proteins into mitochondria: a novel pathomechanism for progressive neurodegeneration. J Inherit Metab Dis 24:166-180.

Bencze KZ, Kondapalli KC, Cook JD, McMahon S, Millán-Pacheco C, Pastor N, Stemmler TL (2006) The structure and function of frataxin. Crit Rev Biochem Mol Biol 41:269-291.

Bradley JL, Blake JC, Chamberlain S, Thomas PK, Cooper JM, Schapira AH (2000) Clinical, biochemical and molecular genetic correlations in Friedreich's ataxia. Hum Mol Genet 9:275-282.

Brookes PS, Yoon Y, Robotham JL, Anders MW, Sheu SS (2004) Calcium, ATP, and ROS: a mitochondrial love-hate triangle. Am J Physiol Cell Physiol 287:C817-C833.

Bruijn LI, Miller TM, Cleveland DW (2004) Unraveling the mechanisms involved in motor neuron degeneration in ALS. Annu Rev Neurosci 27:723-749.

Calabrese V, Lodi R, Tonon C, D'Agata V, Sapienza M, Scapagnini G, Mangiameli A, Pennisi G, Stella AM, Butterfield DA (2005) Oxidative stress, mitochondrial dysfunction and cellular stress response in Friedreich's ataxia. J Neurol Sci 233:145-162.

Chang DT, Reynolds IJ (2006) Mitochondrial trafficking and morphology in healthy and injured neurons. Prog Neurobiol 80:241-268.

Chantrel-Groussard K, Geromel V, Puccio H, Koenig M, Munnich A, Rötig A, Rustin P (2001) Disabled early recruitment of antioxidant defenses in Friedreich's ataxia. Hum Mol Genet 10:2061-2067.

Chen H, Detmer SA, Ewald AJ, Griffin EE, Fraser SE, Chan DC (2003) Mitofusins Mfn1 and Mfn2 coordinately regulate mitochondrial fusion and are essential for embryonic development. J Cell Biol 160:189-200.

Delatycki MB, Camakaris J, Brooks H, Evans-Whipp T, Thorburn DR, Williamson R, Forrest SM (1999) Direct evidence that mitochondrial iron accumulation occurs in Friedreich ataxia. Ann Neurol 45:673-675.

Delatycki MB, Williamson R, Forrest SM (2000) Friedreich ataxia: an overview. J Med Genet 37:1-8.

Delettre C, Lenaers G, Griffoin JM, Gigarel N, Lorenzo C, Belenguer P, Pelloquin L, Grosgeorge J, Turc-Carel C, Perret E, Astarie-Dequeker C, Lasquellec L, Arnaud B, Ducommun B, Kaplan J, Hamel CP (2000) Nuclear gene OPA1, encoding a mitochondrial dynamin-related protein, is mutated in dominant optic atrophy. Nat Genet 26:207-210.

De Vos KJ, Chapman AL, Tennant ME, Manser C, Tudor EL, Lau KF, Brownlees
J, Ackerley S, Shaw PJ, McLoughlin DM, Shaw CE, Leigh PN, Miller CC, Grierson AJ (2007) Familial amyotrophic lateral sclerosis-linked SOD1 mutants perturb fast axonal transport to reduce axonal mitochondria content. Hum Mol Genet 16:2720-2728.

Edgar JM, McLaughlin M, Yool D, Zhang SC, Fowler JH, Montague P, Barrie JA, McCulloch MC, Duncan ID, Garbern J, Nave KA, Griffiths IR (2004) Oligodendroglial modulation of fast axonal transport in a mouse model of hereditary spastic paraplegia. J Cell Biol 166:121-131.

Emond M, Lepage G, Vanasse M, Pandolfo M (2000) Increased levels of plasma malondialdehyde in Friedreich ataxia. Neurology 55:1752-1753.

Estes PS, Ho GL, Narayanan R, Ramaswami M (2000) Synaptic localization and restricted diffusion of a Drosophila neuronal synaptobrevin-green fluorescent protein chimera in vivo. J Neurogenet 13:233-255.

Farkas DL, Wei MD, Febbroriello P, Carson JH, Loew LM (1989) Simultaneous imaging of cell and mitochondrial-membrane potentials. Biophys J 56:1053-1069.

Ferreirinha F, Quattrini A, Pirozzi M, Valsecchi V, Dina G, Broccoli V, Auricchio A, Piemonte F, Tozzi G, Gaeta L, Casari G, Ballabio A, Rugarli EI (2004) Axonal degeneration in paraplegin-deficient mice is associated with abnormal mitochondria and impairment of axonal transport. J Clin Invest 113:231-242.

Floyd RA (1999) Antioxidants, oxidative stress, and degenerative neurological disorders. Proc Soc Exp Biol Med 222:236-245.

Floyd RA, Kotake Y, Hensley K, Nakae D, Konishi Y (2002) Reactive oxygen species in choline deficiency induced carcinogenesis and nitrone inhibition. Mol Cell Biochem 234-235:195-203.

Fukui H, Moraes CT (2008) The mitochondrial impairment, oxidative stress and neurodegeneration connection: reality or just an attractive hypothesis? Trends Neurosci 31:251-256.

Gauthier LR, Charrin BC, Borrell-Pagès M, Dompierre JP, Rangone H, Cordelières FP, De Mey J, MacDonald ME, Lessmann V, Humbert S, Saudou F (2004) Huntingtin controls neurotrophic support and survival of neurons by enhancing BDNF vesicular transport along microtubules. Cell 118:127-138.

Gibson TJ, Koonin EV, Musco G, Pastore A, Bork P (1996) Friedreich’s ataxia protein: phylogenetic evidence for mitochondrial dysfunction. Trends Neurosci 19:465-468.

Gindhart JG Jr, Desai CJ, Beushausen S, Zinn K, Goldstein LS (1998) Kinesin light chains are essential for axonal transport in Drosophila. J Cell Biol 141:443-454.

Glater EE, Megeath LJ, Stowers RS, Schwarz TL (2006) Axonal transport of mitochondria requires milton to recruit kinesin heavy chain and is light chain independent. J Cell Biol 173:545-557.

Guo X, Macleod GT, Wellington A, Hu F, Panchumarthi S, Schoenfield M, Marin L, Charlton MP, Atwood HL, Zinsmaier KE (2005) The GTPase dMiro is required for axonal transport of mitochondria to Drosophila synapses. Neuron 47:379-393.

Hafezparast M, Klocke R, Ruhrberg C, Marquardt A, Ahmad-Annuar A, Bowen S, Lalli G, Witherden AS, Hummerich H, Nicholson S, Morgan PJ, Oozageer R, Priestley JV, Averill S, King VR, Ball S, Peters J, Toda T, Yamamoto A, Hiraoka Y, et al. (2003) Mutations in dynein link motor neuron degeneration to defects in retrograde transport. Science 300:808-812.

Halliwell B (2006) Oxidative stress and neurodegeneration: where are we now? J Neurochem 97:1634-1658.

Hirokawa N, Takemura R (2004) Molecular motors in neuronal development, intracellular transport and diseases. Curr Opin Neurobiol 14:564-573.

Hollenbeck PJ, Saxton WM (2005) The axonal transport of mitochondria. J Cell Sci 118:5411-5419.

Horiuchi D, Barkus RV, Pilling AD, Gassman A, Saxton WM (2005) APLIP1, a kinesin binding JIP-1/JNK scaffold protein, influences the axonal transport of both vesicles and mitochondria in Drosophila. Curr Biol 15:2137-2141.

Hurd DD, Saxton WM (1996) Kinesin mutations cause motor neuron disease phenotypes by disrupting fast axonal transport in Drosophila. Genetics 144:1075-1085.

Hurd DD, Stern M, Saxton WM (1996) Mutation of the axonal transport motor kinesin enhances paralytic and suppresses Shaker in Drosophila. Genetics 142:195-204

Ibáñez CF (2007) Message in a bottle: long-range retrograde signaling in the nervous system. Trends Cell Biol 17:519-528. 
Jiralerspong S, Ge B, Hudson TJ, Pandolfo M (2001) Manganese superoxide dismutase induction by iron is impaired in Friedreich ataxia cells. FEBS Lett 509:101-105.

Keshishian H, Kim YS (2004) Orchestrating development and function: retrograde BMP signaling in the Drosophila nervous system. Trends Neurosci $27: 143-147$.

Kijima K, Numakura C, Izumino H, Umetsu K, Nezu A, Shiiki T, Ogawa M, Ishizaki Y, Kitamura T, Shozawa Y, Hayasaka K (2005) Mitochondrial GTPase mitofusin 2 mutation in Charcot-Marie-Tooth neuropathy type 2A. Hum Genet 116:23-27.

LaMonte BH, Wallace KE, Holloway BA, Shelly SS, Ascaño J, Tokito M, Van Winkle T, Howland DS, Holzbaur EL (2002) Disruption of dynein/dynactin inhibits axonal transport in motor neurons causing late-onset progressive degeneration. Neuron 34:715-727.

Liu Z, Butow RA (2006) Mitochondrial retrograde signaling. Annu Rev Genet 40:159-185.

Llorens JV, Navarro JA, Martínez-Sebastián MJ, Baylies MK, Schneuwly S, Botella JA, Moltó MD (2007) Causative role of oxidative stress in a Drosophila model of Friedreich ataxia. FASEB J 21:333-344.

Lodi R, Cooper JM, Bradley JL, Manners D, Styles P, Taylor DJ, Schapira AH (1999) Deficit of in vivo mitochondrial ATP production in patients with Friedreich ataxia. Proc Natl Acad Sci U S A 96:11492-11495.

Lu C, Cortopassi G (2007) Frataxin knockdown causes loss of cytoplasmic iron-sulfur cluster functions, redox alterations and induction of heme transcripts. Arch Biochem Biophys 457:111-122.

Maher P, Schubert D (2000) Signaling by reactive oxygen species in the nervous system. Cell Mol Life Sci 57:1287-1305.

Marqués G, Zhang B (2006) Retrograde signaling that regulates synaptic development and function at the Drosophila neuromuscular junction. Int Rev Neurobiol 75:267-285.

McCabe BD, Marqués G, Haghighi AP, Fetter RD, Crotty ML, Haerry TE, Goodman CS, O'Connor MB (2003) The BMP homolog Gbb provides a retrograde signal that regulates synaptic growth at the Drosophila neuromuscular junction. Neuron 39:241-254.

McFarland R, Taylor RW, Turnbull DM (2007) Mitochondrial disease-its impact, etiology, and pathology. Curr Top Dev Biol 77:113-155.

McKenzie M, Liolitsa D, Hanna MG (2004) Mitochondrial disease: mutations and mechanisms. Neurochem Res 29:589-600.

Mukhopadhyay P, Rajesh M, Haskó G, Hawkins BJ, Madesh M, Pacher P (2007) Simultaneous detection of apoptosis and mitochondrial superoxide production in live cells by flow cytometry and confocal microscopy. Nat Protoc 2:2295-2301.

Patel PI, Isaya G (2001) Friedreich ataxia: from GAA triplet-repeat expansion to frataxin deficiency. Am J Hum Genet 69:15-24.

Piemonte F, Pastore A, Tozzi G, Tagliacozzi D, Santorelli FM, Carrozzo R, Casali C, Damiano M, Federici G, Bertini E (2001) Glutathione in blood of patients with Friedreich's ataxia. Eur J Clin Invest 31:1007-1011.

Pilling AD, Horiuchi D, Lively CM, Saxton WM (2006) Kinesin-1 and Dynein are the primary motors for fast transport of mitochondria in Drosophila motor axons. Mol Biol Cell 17:2057-2068.

Pitkanen S, Robinson BH (1996) Mitochondrial complex I deficiency leads to increased production of superoxide radicals and induction of superoxide dismutase. J Clin Invest 98:345-351.

Puccio H, Koenig M (2002) Friedreich ataxia: a paradigm for mitochondrial diseases. Curr Opin Genet Dev 12:272-277.

Puccio H, Simon D, Cossée M, Criqui-Filipe P, Tiziano F, Melki J, Hindelang C, Matyas R, Rustin P, Koenig M (2001) Mouse models for Friedreich ataxia exhibit cardiomyopathy, sensory nerve defect and Fe-S enzyme deficiency followed by intramitochondrial iron deposits. Nat Genet 27:181-186.

Puls I, Jonnakuty C, LaMonte BH, Holzbaur EL, Tokito M, Mann E, Floeter MK, Bidus K, Drayna D, Oh SJ, Brown RH Jr, Ludlow CL, Fischbeck KH (2003) Mutant dynactin in motor neuron disease. Nat Genet 33:455-456.

Rötig A, de Lonlay P, Chretien D, Foury F, Koenig M, Sidi D, Munnich A, Rustin P (1997) Aconitase and mitochondrial iron-sulphur protein deficiency in Friedreich ataxia. Nat Genet 17:215-217.

Runko AP, Griswold AJ, Min KT (2008) Overexpression of frataxin in the mitochondria increases resistance to oxidative stress and extends lifespan in Drosophila. FEBS Lett 582:715-719.

Sanyal S, Kim SM, Ramaswami M (2004) Retrograde regulation in the CNS; neuron-specific interpretations of TGF-beta signaling. Neuron $41: 845-848$

Saxton WM, Hicks J, Goldstein LS, Raff EC (1991) Kinesin heavy chain is essential for viability and neuromuscular functions in Drosophila, but mutants show no defects in mitosis. Cell 64:1093-1102.

Scaduto RC, Grotyohann LW (1999) Measurement of mitochondrial membrane potential using fluorescent rhodamine derivatives. Biophys J 76:469-477.

Schapira AH (2006) Mitochondrial disease. Lancet 368:70-82.

Schulz JB, Dehmer T, Schöls L, Mende H, Hardt C, Vorgerd M, Bürk K, Matson W, Dichgans J, Beal MF, Bogdanov MB (2000) Oxidative stress in patients with Friedreich ataxia. Neurology 55:1719-1721.

Seznec H, Simon D, Monassier L, Criqui-Filipe P, Gansmuller A, Rustin P, Koenig M, Puccio H (2004) Idebenone delays the onset of cardiac functional alteration without correction of Fe-S enzymes deficit in a mouse model for Friedreich ataxia. Hum Mol Genet 13:1017-1024.

Seznec H, Simon D, Bouton C, Reutenauer L, Hertzog A, Golik P, Procaccio V, Patel M, Drapier JC, Koenig M, Puccio H (2005) Friedreich ataxia: the oxidative stress paradox. Hum Mol Genet 14:463-474.

Smeitink JA, van den Heuvel LW, Koopman WJ, Nijtmans LG, Ugalde C, Willems PH (2004) Cell biological consequences of mitochondrial NADH: ubiquinone oxidoreductase deficiency. Curr Neurovasc Res $1: 29-40$.

Staniek K, Nohl H (2000) Are mitochondria a permanent source of reactive oxygen species? Biochim Biophys Acta 1460:268-275.

Stehling O, Elsässer HP, Brückel B, Mühlenhoff U, Lill R (2004) Iron-sulfur protein maturation in human cells: evidence for a function of frataxin. Hum Mol Genet 13:3007-3015.

Stowers RS, Megeath LJ, Górska-Andrzejak J, Meinertzhagen IA, Schwarz TL (2002) Axonal transport of mitochondria to synapses depends on milton, a novel Drosophila protein. Neuron 36:1063-1077.

St-Pierre J, Buckingham JA, Roebuck SJ, Brand MD (2002) Topology of superoxide production from different sites in the mitochondrial electron transport chain. J Biol Chem 277:44784-44790.

Taylor RW, Turnbull DM (2005) Mitochondrial DNA mutations in human disease. Nat Rev Genet 6:389-402.

Verburg J, Hollenbeck PJ (2008) Mitochondrial membrane potential in axons increases with local nerve growth factor or semaphorin signaling. J Neurosci 28:8306-8315.

Verhoeven K, Claeys KG, Züchner S, Schröder JM, Weis J, Ceuterick C, Jordanova A, Nelis E, De Vriendt E, Van Hul M, Seeman P, Mazanec R, Saifi GM, Szigeti K, Mancias P, Butler IJ, Kochanski A, Ryniewicz B, De Bleecker J, Van den Bergh P, et al. (2006) MFN2 mutation distribution and genotype/phenotype correlation in Charcot-Marie-Tooth type 2. Brain 129:2093-2102.

Verstreken P, Ly CV, Venken KJ, Koh TW, Zhou Y, Bellen HJ (2005) Synaptic mitochondria are critical for mobilization of reserve pool vesicles at Drosophila neuromuscular junctions. Neuron 47:365-378.

Wallace DC (2005) A mitochondrial paradigm of metabolic and degenerative diseases, aging, and cancer: a dawn for evolutionary medicine. Annu Rev Genet 39:359-407.

Wilson RB, Roof DM (1997) Respiratory deficiency due to loss of mitochondrial DNA in yeast lacking the frataxin homologue. Nat Genet 16:352-357.

Wong A, Yang J, Cavadini P, Gellera C, Lonnerdal B, Taroni F, Cortopassi G (1999) The Friedreich's ataxia mutation confers cellular sensitivity to oxidant stress which is rescued by chelators of iron and calcium and inhibitors of apoptosis. Hum Mol Genet 8:425-430.

Yonekawa Y, Harada A, Okada Y, Funakoshi T, Kanai Y, Takei Y, Terada S, Noda T, Hirokawa N (1998) Defect in synaptic vesicle precursor transport and neuronal cell death in KIF1A motor protein-deficient mice. J Cell Biol 141:431-441.

Zhao C, Takita J, Tanaka Y, Setou M, Nakagawa T, Takeda S, Yang HW, Terada S, Nakata T, Takei Y, Saito M, Tsuji S, Hayashi Y, Hirokawa N (2001) Charcot-Marie-Tooth disease type 2A caused by mutation in a microtubule motor KIF1Bbeta. Cell 105:587-597. 